\title{
Realistic Mobility Simulation of Urban Mesh Networks
}

\author{
Jonghyun Kim Vinay Sridhara Stephan Bohacek* \\ Department of Electrical and Computer Engineering \\ University of Delaware \\ Newark, DE 19716
}

\begin{abstract}
It is a truism that today's simulations of mobile wireless networks are not realistic. In realistic simulations of urban networks, the mobility of vehicles and pedestrians is greatly influenced by the environment (e.g., the location of buildings) as well as by interaction with other nodes. For example, on a congested street or sidewalk, nodes cannot travel at their desired speed. Furthermore, the location of streets, sidewalks, hallways, etc. restricts the position of nodes, and traffic lights impact the flow of nodes. And finally, people do not wander the simulated region at random, rather, their mobility depends on whether the person is at work, at lunch, etc. In this paper, realistic simulation of mobility for urban wireless networks is addressed. In contrast to most other mobility modeling efforts, most of the aspects of the presented mobility model and model parameters are derived from surveys from urban planning and traffic engineering research. The mobility model discussed here is part of the UDel Models, a suite of tools for realistic simulation of urban wireless networks. The UDel Models simulation tools are available online.
\end{abstract}

Key words: urban mesh networks, simulation, mobility, mobile wireless networks

\section{Introduction}

By providing connectivity to mobile users, mesh networks are poised to become a major extension of the Internet. More than 300 cities and towns have plans to deploy mesh networks, and several dozen cities have already deployed mesh networks [1]. While some deployments have been in smaller cities, such as Mountain View, CA and St. Cloud, FL. Some deployments

* Corresponding author. Address: Department of Electrical and Computer Engineering University of Delaware Newark, DE 19716 Phone: 302-831-4274 Fax: 302-831-4316 Email: bohacek@udel.edu 
have been in larger cities such as Corpus Christi's 147 sq. mile deployment [2]. Furthermore, large cities such as Philadelphia [3] and San Francisco [4] are in the final planning stages of city-wide, dense deployments. These mesh networks are meant to enhance city and emergency services communication as well as to provide city-wide, low-cost, ubiquitous Internet access for residents and visitors. Such networks promise to bring dramatic changes to data accessibility and hence have a major impact on society.

While mesh networks have much promise, there are important issues regarding performance and scalability that have yet to be resolved. However, developing new protocols for large-scale urban mesh networks (LUMNets) is stymied by the lack of realistic simulators for testing protocol performance. The simulation of LUMNets is quite different from MANETs (as well as other mobile wireless networks) in that the environment plays an important role in the performance of LUMNets. For example, the layout of the city determines which nodes can communicate, and streets form propagation conduits. Furthermore, urban mobility is quite distinct from mobility for general purpose or military MANETs.

While simulation of mobile wireless networks has long been a difficult problem, the influence on propagation and mobility on LUMNet performance requires new efforts in simulation. To further motivate the need for mobility and propagation simulation, consider the problem of mobility management for LUMNets (which is necessary for scalability). As is the case for mobile phone networks $[5,6,7,8,9]$, there are many mobility management techniques that could be applied to LUMNets. However, the performance of these schemes is greatly influenced by node mobility and the propagation range of base stations. For example, small indoor coverage areas, may result in rapid node migration, whereas large outdoor coverage areas results in slower node migration when the node is a walking person, but more rapid migration when the person is in a car. Mobility management is further complicated by the fact that some base stations will have coverage that extends both indoors and outdoors. See [10] for an example where the propagation characteristics of an urban area are exploited for efficient mobility management. Realistic propagation and mobility are not only required for performance evaluation of mobility management, but are also required for the performance evaluation of routing, transport, scheduling, etc.

While realistic propagation has previously been examined [11], realistic mobility has received less attention. The approach to realistic mobility models described here is significantly different from other mobility models in that much of the model is based on surveys. Specifically, the simulator uses surveys on time-use from the US Bureau of Labor Statistics, and an extensive set of surveys of pedestrian and vehicle mobility developed within Urban Planning (e.g., $[12,13])$. Furthermore, to determine mobility within office buildings, surveys from the meetings analysis research area are used. It should be stressed, that the mobility model is not ad hoc, but based on the findings of mature research communities. For example, TimeUse Studies has been active for approximately 40 years [14] and many aspects of the agent mobility (see Section 4 for definition), have been known for 30 years and are integrated into government guidelines on traffic planning [13]. This paper distills the results of these areas and presents the aspects that are important for urban mobility. 
The realistic mobility model discussed here is comprehensive in that many different types of urban mobility are supported. Of course, if only one type of mobility is of interest, it is not necessary to simulate all types of mobility. For example, the mobility model discussed here is implemented and freely available [15], and while the implementation provides mobility for many types of urban mobility, it is possible to focus on specific types of mobility (see Section 6 for some examples).

The remainder of the paper proceeds as follows. In the next section, an overview of the simulation of urban networks is presented. Section 3 discusses techniques for developing city maps. Clearly, mobility and propagation are greatly effected by the map. Section 4 presents the mobility model of people. This model has three parts, namely, the activity model, the task model, and the agent model. These models are discussed in subsections 4.1, 4.3, and 4.4, respectively. Subsection 4.5 provides some details on how commuting is implemented, while subsection 4.6 discusses how realistic population sizes can be determined. Section 5 presents a model for car mobility. Section 6 investigates the impact that realistic mobility models have on network performance. Related work on mobility modeling is provided in Section 7. Then future directions in realistic mobility modeling are discussed in Section 8 and concluding remarks are made in Section 9.

\section{Mobile Wireless Network Simulation Overview}

There are several stages to LUMNet simulation. The first step is to define the simulated city map. This step is discussed in Section 3. The second step is to determine the propagation matrix for the simulated region. The propagation matrix includes characteristics such as the channel gain, delay spread, and angle of arrival for each possible transmitter-receiver pair in the simulated region. Simulating urban propagation is discussed in [11]. Next, the city map is used to generate one or more mobility trace files. Realistic urban mobility is the focus of this paper. From the mobility trace file and the propagation matrix, the propagation trace file is computed; the propagation trace file provides the channel model between all pairs of nodes at every moment of the simulation. The propagation trace file can then be used by a protocol simulator such as QualNet, ns-2, or OPNET.

\section{$3 \quad$ City Maps}

In order to simulate an urban wireless network, it is necessary to have a model of the urban area. There are several ways that maps for simulation can be developed. First, a random city can be built as was done in [16]. In this case, buildings are placed at random and a Voronoi diagram is used to construct sidewalks between the buildings. One drawback of such an approach is that important aspects of cities such as long thoroughfares and big intersections are neglected. It is well known that streets play an important role in mobile phone 


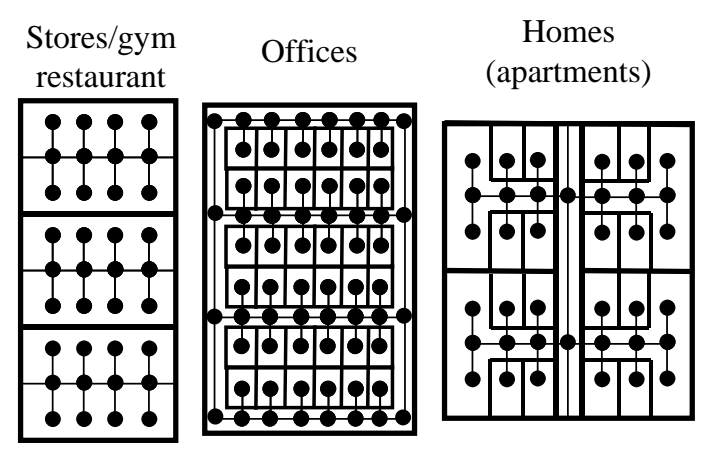

Fig. 1. Locations in different types of buildings. Locations are marked with a circle while arcs are indicated by thin lines. The thick lines denote walls. The store/gym/restaurant structure is such that each third of the layout can be any of these options. The apartment building shown has four apartments each with five rooms. The size and number of rooms depends on the building size.

communication and it has been shown that streets play an important role in connectivity in MANETs [17].

A more realistic way to generate cities is to utilize detailed GIS data sets [18]. These data sets include 3-dimensional maps of buildings that provide enough detail for realistic simulation. There is a large number of such data sets. For example, there are GIS data sets for most American cities. The UDel Models map building suite of tools converts GIS data sets into format suitable for a specialized graphical editor. The graphical editor is used to "touchup" the GIS map (e.g., remove spurious buildings). The editor is also used to add roads, sidewalks, traffic lights, base stations, subway stations, define the types of buildings (e.g., residence, store/restaurant, office), and define building materials (building materials impact propagation [11]). While GIS data sets have details of building heights and position, they typically do not provide details about the interiors of the building. In lieu of actual interiors, they must be automatically generated. The UDel Models uses layouts shown in Figure 1.

Another realistic method to generate city maps is to use US Census Bureau's TIGER data (Topologically Integrated Geographic Encoding and Referencing) [19]. The TIGER data includes roads, railroads, rivers, lakes, and legal boundaries in the US. It also contains information about roads including their location in latitude and longitude, name, type, address ranges, and speed limits. However, it does not include information about buildings. TIGER data is often used for realistic maps for simulating vehicle ad hoc networks $[20,21,22]$.

In general, nodes (people or vehicles) may be at a large number of locations within the city. However, a significant computational savings are achieved if the nodes are restricted to a specific graph. The UDel Models define a large set of locations (vertices) and pathways (arcs). The nodes are restricted to move along this graph. Examples of parts of this graph are shown in Figure 1. 


\section{Mobility of People}

This section presents a detailed mobility model of urban pedestrians during the workday. This model is based on three mature research areas, namely, urban planning [12, 13], meeting analysis [23], and use of time [14]. The resulting model is a three layer hierarchical model. The top layer is the activity model that determines high-level types of activities, the time when people start and end the activities as well as the location where the activity is performed. The data used to develop this model is from the 2003 US Bureau of Labor Statistics (BLS) use of time study [24]. This study includes interviews with roughly 20,000 people. Furthermore, the BLS determined weightings to account for oversampling of some types of people (e.g., unemployed people tend to be at home at the time of the interview call and tend to be oversampled). Hence, the significance of the study exceeds the 20,000 that were actually interviewed. This study collected detailed data on the interviewee's day included the times that activities were started and stopped, where the activities were performed, and for what reason the activity was performed.

The second layer of the pedestrian mobility model is the task model. While performing a particular activity, a person may carry out many tasks. For example, the model discussed here focuses on office workers. While such nodes are performing a work activity, there are two possible tasks, namely, working at their desk, and meeting with other workers. The basis of this part of the mobility model is several seminal studies of worker meetings performed within the management research community (see [23] and references therein). This part of the model allows one to determine how nodes move within a building and how nodes are clustered within buildings. Mobility within buildings is important if networks utilize relaying by mobile nodes. For example, an outdoor network such as Philadelphia's can greatly increase its indoor coverage if mobile nodes can act as relays [25]. To determine the performance of such relaying, the mobility of indoor nodes must be modeled.

The third layer of the mobility model is the agent model. Such agent models have been investigated within the architecture community and define how nodes navigate walkways to their desired destinations. This model is based on urban planning research, especially the seminal work of Pushkarev and Zupan [12] as well as several other pedestrian mobility studies. A key feature of this part of the mobility model is that it realistically models how nodes form clusters or platoons. Such clusters are important since nodes in close proximity will experience strong interference. On the other hand, the formation of ad hoc or virtual antenna arrays can be enhanced by the presence of clusters of nodes. For these reasons, the model includes several mechanisms that impact platooning.

\subsection{Activity Model}

This part of the mobility model is based on the US Bureau of Labor Statistics (BLS) 2003 time-use study [24]. This study identifies a large number of activities. We focus on those activities that indicate location, and group together activities that are performed in the 
same location (e.g., all activities performed at home are grouped together into the at home activity). While the BLS study also collected coarse location information, both activity and location information were used to determine the location used in the modeling effort. We focus on eight types of activities: working, eating not at work, shopping, at home, receiving professional service, exercise, relaxing, and dropping off someone. Note that since we focus on location and mobility, eating at work is counted as work. Eating not at work includes eating at a restaurant and buying food somewhere besides at work. Shopping includes all types of shopping except buying food. Receiving professional service ranges from things such as getting medical attention to receiving household management and maintenance services that are not performed at home.

During the simulation initialization, each node is given an office and home. It is assumed that work is done within the building where the nodes office is located (work done at home is included into the at home activity). Eating is done at a restaurant (eating at home is included into at home activity). Shopping is done at a randomly selected store. Receiving professional service is done at an office that is not the node's office. We do not specify special locations for relaxing or dropping someone off. Dropping someone off includes meeting children at school and taking them home. For the purpose of mobility modeling, we model such activities as a trip home followed by a trip to a random selected office location. The node remains at the office location until the drop off activity if complete. The relaxing activity is modeled as going to an office location (much like receiving professional service).

This model focuses on the work day which consists of being at home, going to work, working, perhaps taking a break, leaving work, and returning home. The model neglects activities before and after work. Future work will include the rest of the day.

For each person, the following steps are taken to determine the activities that they perform.

(1) Select a home and office.

(2) Determine the arrival time at work.

(3) Determine the duration at work.

(4) Determine if a break from work is taken. (The next 5 steps assume a break is taken.)

(5) Determine the break start time.

(6) Determine the number of activities performed during a break

(7) Determine which activities are performed during the break.

(8) Determine the duration of each activity.

(9) Determine the arrival time back at work and determined if a break is taken again. If so, steps 5-9 are repeated.

Selection of home and office For each simulated person, an office is selected at random. Once an office is selected, a home is selected that is nearby the office. In case the person does not live in the city (the fraction of people that live within the city depends on the amount of residential area), then the person enters the city by subway or by car. In such cases, instead of assigning the person a home, they are assigned a parking-lot or a subway stop. The home, parking-lot, and subway stops are selected so that the distance to the office matches the distribution shown in Figure 7. This distribution is based on walking distances 
Table 1

\begin{tabular}{|l||l|l|l|l|}
\hline time & $\alpha$ & $\mu$ & $\sigma$ & $m$ \\
\hline$\leq 8 \mathrm{AM}$ & 0.91 & $8: 09$ & $1: 06$ & $9: 50$ \\
\hline $8-9$ & 0.85 & $7: 49$ & $0: 56$ & $8: 52$ \\
\hline $9-10$ & 0.81 & $7: 16$ & $1: 17$ & $5: 52$ \\
\hline $10-11$ & 1.0 & $7: 11$ & $2: 16$ & - \\
\hline $11-12$ & 0.70 & $7: 16$ & $2: 11$ & $5: 00$ \\
\hline $12-1$ & 1.0 & $6: 19$ & $2: 40$ & - \\
\hline $1-3$ & 0.5 & $7: 33$ & $0: 55$ & $4: 31$ \\
\hline $3-6$ & 0.83 & $6: 18$ & $1: 55$ & $2: 07$ \\
\hline$\geq 6$ & 1.0 & $4: 30$ & $2: 26$ & - \\
\hline
\end{tabular}

Duration at work model parameters

observed by Pushkarev and Zupan. Further discussion on the mode of travel to work can be found in Section 4.5.

Arrival time at work Figure 2 shows the complementary cumulative distribution function $(\mathrm{CCDF})$ of the time of arrival at work. The observed values were fitted with a mixture of exponential and Gaussian distribution. Specifically, with probability of 0.552 , the time of arrival is normally distributed with mean 7:46 a.m. and standard deviation of 45 minutes. With probability $(1-0.552)$, the time of arrival is exponentially distributed with the mean time of arrival of 12:00. The exponential distribution is shifted so that the earliest minimum time of arrival in this case is 5AM. Similarly, the normal distribution is truncated so that no arrivals occur before 5AM.

Duration at work Figure 3 shows the CCDF of the duration at work for people that arrive at work between 7 and 8 in the morning and for those that arrive between 10 and 11 in the morning. These distributions and ones for other arrival times at work were fitted with a mixture of a normal random variable and an exponential random variable. These distributions have four parameters, $\alpha$, the probability of selecting the normal distribution, $\mu$ and $\sigma$ the mean and the standard deviation of the normal distribution and $m$, the mean of the exponential distribution. Table 1 shows the value of these parameters for the different arrival times at work. Surprisingly, while the model is simple, the fit shown in Figure 3 is a typical quality of fit throughout the day. On the other hand, from Figure 2 it can be seen that the most important distribution is that for nodes arriving between 7 and 8 .

Whether a break is taken The probability of whether a break is taken depends on the time of arrival at work. Note that if a break is not taken, the person may still eat lunch, but they do not leave the building. We fit the probability of taking a break given the time 


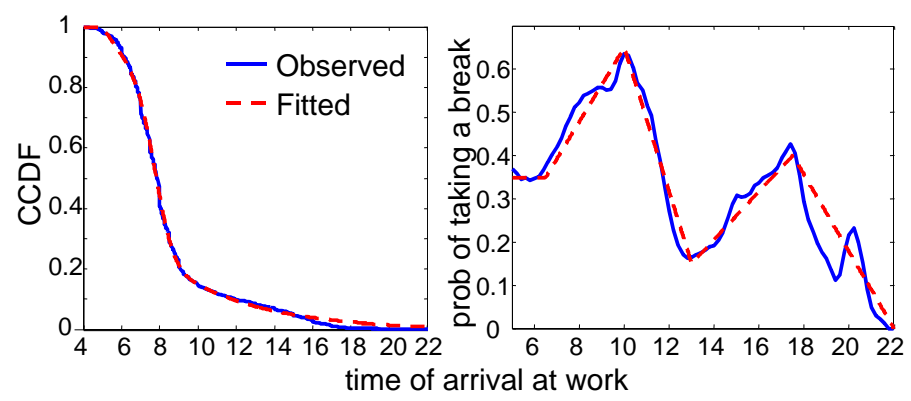

Fig. 2. Left. The complimentary cumulative distribution function (CCDF) of the time of arrival at work. Right. The probability of taking a break given the arrival time at work.

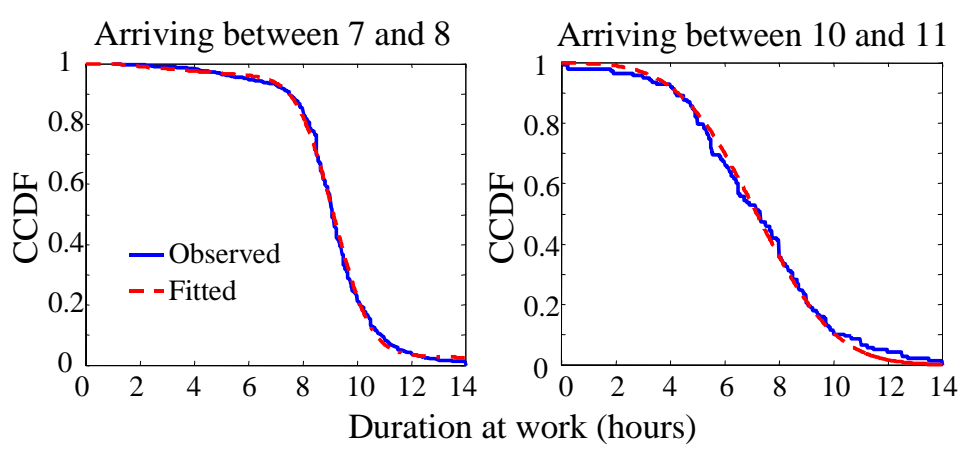

Fig. 3. The CCDF of the duration at work for two different arrival times at work.

of arrival with a piece-wise linear function.

$$
P(\text { taking a break } \mid \text { arrival time at work }=t)=\left\{\begin{array}{c}
0.35 \text { for } t<6.5 \\
0.86(t-6.5)+0.35 \text { for } 6.5 \leq t \leq 10 \\
0.17(t-10)-0.65 \text { for } 10 \leq t \leq 13 \\
0.056(t-13)+0.15 \text { for } 13 \leq t \leq 17.5 \\
-0.08(t-17.50)+0.4 \text { for } t \geq 17.5
\end{array}\right.
$$

Note that this equation uses fraction of hours past midnight, not hours and minutes. This model and the observed probability is shown in Figure 2.

The time the break is started Clearly one cannot go on a break before they arrive at work. However, once they arrive at work, the rate that a person goes on a break does not significantly depend on how long they have been at work. Figure 4 shows this rate conditioned on the person arriving at work one hour ago, two hours ago, and unconditionally. It can be seen that the duration at work has only a minor impact on the time to take a break and that this difference is within the confidence intervals. Thus, we assume that the rate of going on a break is independent of arrival time, assuming that the node has already arrived at work. 
The rate that a person takes a break is approximated by

$$
r(t)=\left\{\begin{array}{rl}
0.004 \text { for } t<10.5 \\
0.006 \times \exp (-1.7(12-t)) & \text { for } 10.5 \leq 12 \\
0.006 \times \exp (-0.6(t-12)) & \text { for } 12 \leq t \leq 14 \\
0.0058 \times \exp (-0.3(5-t)) & \text { for } 14 \leq t \leq 18 \\
0.0058 \text { for } t>18
\end{array} .\right.
$$

By rate of taking a break, we mean that the probability that a node will take a break within the time interval from $t_{0}$ to $t_{1}$ is $\left(t_{1}-t_{0}\right) \int_{t_{0}}^{t_{1}} r(\tau) d \tau$.

Number of activities performed during a break Figure 5 shows the probability of performing different numbers of activities during a break. We see that over the course of the day, the number of activities performed varies. However, the variation is small, and hence we model the probability to be independent of the time of day.

Which activities are performed during a break The types of activities performed during a break strongly depend on the number of activities to be performed. Figure 5 shows the fraction of breaks that include the indicated activity. Note that if more than one activity is performed, the fractions sum to more than one.

The duration of activities The time spent performing an activity depends on the type of activity. Figure 6 shows the CCDF of the duration of three activities. The distribution of the duration of eating shows a jump at 1 hour. Smaller jumps are noticeable in the distribution of other activities. The duration of these and the other activities are modeled as a mixture of an exponentially distributed random variable conditioned on the duration being larger than a minimum duration along with deterministic duration of one hour. Thus, the distribution of the duration of each activity has three parameters, $\mu$, the mean of the exponential distribution, $\underline{d}$, the minimum duration, and $\rho$, the probability of the duration lasting exactly one hour. Table 2 shows the values of the model parameters for the different activities considered.

Once the activity has been selected, the location of the activity must be determined. Specifically, eating requires selecting a restaurant, exercising requires selecting a gym, getting professional service requires selecting an office location, shopping requires selecting a store, dropping someone off requires selecting an office location to drop them off at. We assume that people walk to the location that is required to perform the activity. Future work will include the case where people take other forms of transportation. Pushkarev and Zupan [12] observed the distribution of the distance that pedestrians walk (see Figure 7). We see that the distance is well modeled by an exponential distribution with means $554 \mathrm{~m}, 380 \mathrm{~m}, 403 \mathrm{~m}$, $344 \mathrm{~m}, 813 \mathrm{~m}$, and $216 \mathrm{~m}$ for Manhattan from office buildings, Manhattan from residences, Chicago, Seattle, London and Edmonton respectively. We see that the US cities have approximately the same mean. Thus, we select a location of the correct type (e.g., a store for shopping) at random such that the walking distance is exponentially distributed with mean 
Table 2

\begin{tabular}{|l|l|l|l|}
\hline activity & $\mu$ & $\underline{\mathrm{d}}$ & $\rho$ \\
\hline eat & $0: 31$ & $0: 20$ & 0.18 \\
\hline shop & $0: 28$ & $0: 20$ & 0.03 \\
\hline at home & $1: 00$ & $0: 20$ & 0.12 \\
\hline professional & $0: 44$ & $0: 10$ & 0.04 \\
\hline exercise & $0: 35$ & $0: 20$ & 0 \\
\hline relax & $0: 27$ & $0: 15$ & 0.01 \\
\hline drop-off & $0: 19$ & $0: 10$ & 0.02 \\
\hline
\end{tabular}

Duration of activity model parameters

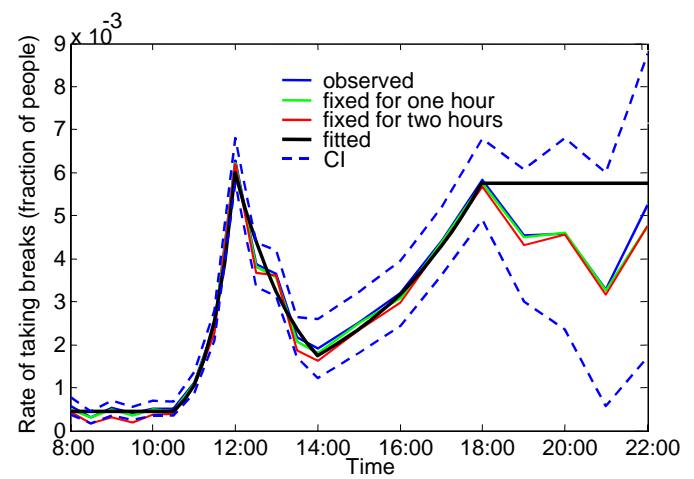

Fig. 4. The rate that a person takes a break and leaves work given the current time. Also shown are the rates conditioned on the person being at work for at least one and two hours. These rates are within the confidence intervals that are also shown. Finally, the fitted rate is also shown.
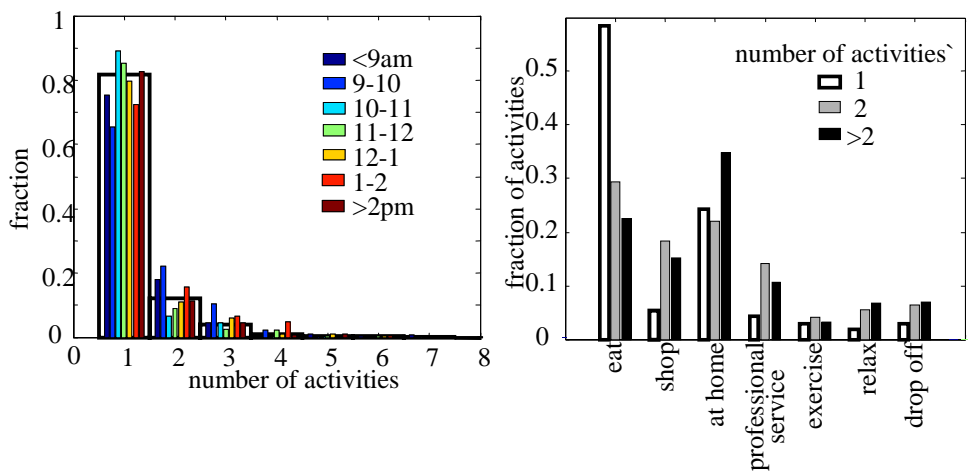

Fig. 5. Left: the number of activities done during a break conditioned on the time that the break is started. Right: the fraction of time that a break includes the indicated activity given the number of activities performed within the break.

$400 \mathrm{~m}$. 


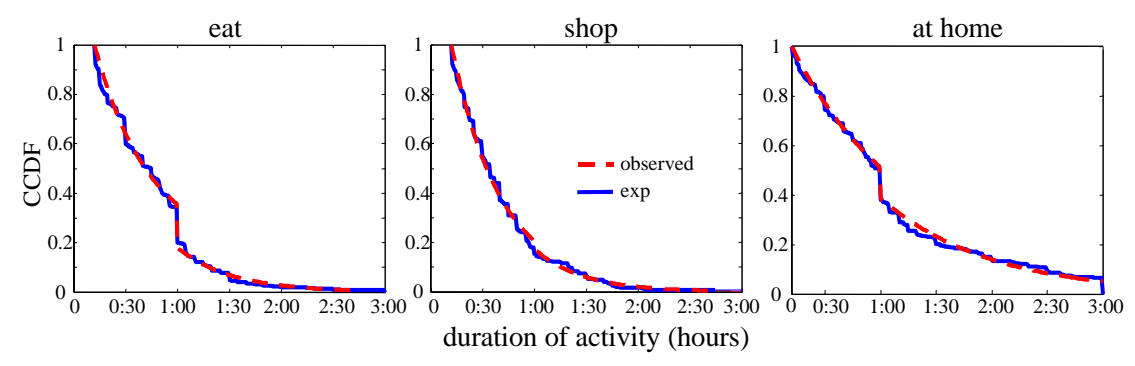

Fig. 6. CCDF of the duration of eat, shop, and at home activities.

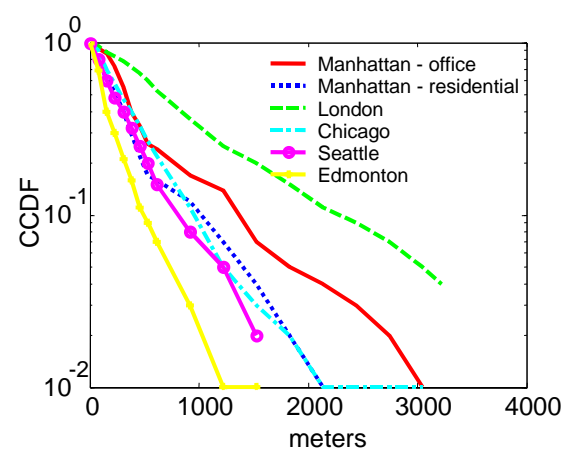

Fig. 7. CCDF of Distance Traveled During Outdoor Walking Trips. This data is from [12].

\subsection{Activity model of people who did not work}

On a particular work day, about $8 \%$ of people interviewed by the BLS did not work. While these people have a wide variety of activities, an approximate model is as follows. With a probability of 0.54 , a sequence of trips is started at a time with the same distribution as the arrival time to work given above. Upon arriving at the desired destination (which we model as a random office in the region), the time that the person remains at the location is exponentially distributed mean 56 minutes. Then, with probability 0.37 , the person begins another trip, and with probability 0.63 , the person returns home. A second sequence is trips is started with probability 0.72 at a time that is normally distributed with mean 14:27 and standard deviation of 118 minutes. These sequence of trips has the same distribution as the morning trips.

\subsection{Task Model}

Some activities consist of a single task. For example, eating consists of going to a restaurant. However, shopping and working consist of multiple tasks. We model shopping as a simple random walk inside the store. However, this model is based on intuition; future work is required to verify this model. The work activity is modeled in a more complicated manner that focuses on modeling meetings. Specifically, [23, 26, 27] have collected data on the frequency, size, and durations of meetings; [26] includes two person meetings. These studies allow the model to include worker interactions. Thus, we model mobility while at work as a sequence 
Table 3

\begin{tabular}{|l|l|l|}
\hline meeting size & mean duration & prob. \\
\hline 2 & $21(\mathrm{~min})$ & 0.65 \\
\hline 3 & 19 & 0.12 \\
\hline 4 & 57 & 0.04 \\
\hline 5 & 114 & 0.02 \\
\hline 6 & 37 & 0.04 \\
\hline 7 & 50 & 0.03 \\
\hline 8 & 150 & 0.01 \\
\hline 9 & 75 & 0.02 \\
\hline 10 & 150 & 0.01 \\
\hline 15 & 30 & 0.025 \\
\hline 20 & 30 & 0.025 \\
\hline
\end{tabular}

Meetings model parameters

of meetings followed by working in the node's office. This process repeats until the work activity is complete.

More specifically, meetings are simulated as follows. The time between meetings is assumed to be exponentially distributed. When a meeting begins, a random number of people are selected to attend the meeting. Based on the number of people attending, the duration of the meeting is determined. The duration is assumed to be exponentially distributed.

The model parameters of the model are the mean time between meetings, the distribution of the size of meetings, and the relationship between number of meeting participants and the mean meeting duration. These parameters are derived from [23, 26, 27]. Specifically, the mean time between meetings is 18 minutes while Table 3 gives the remaining of the model parameters.

\subsection{Agent Model - Node Dynamics and Interactions}

Since the pioneering work of Pushkarev and Zupan [12], it has been known that pedestrians are not uniformly distributed but tend to be group into clusters or, in the terminology of urban planning, platoons. Since the distribution of nodes plays an important role in the performance of mesh networks, the mobility must also model platoons. This part of the model is known as the agent model and is responsible for determining the trajectory of the node as it moves from one location to the next. It is assumed that nodes take the shortest path, hence path finding is not an important part of the agent model. Rather, the agent model focuses on the dynamics and interaction between moving nodes. More specifically, 


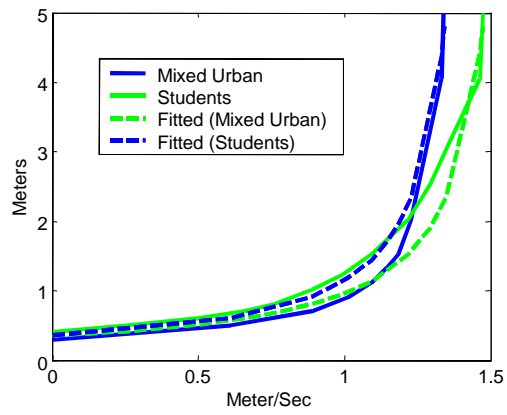

Fig. 8. Speed-distance relationship for pedestrians. The mixed urban pedestrian data is adapted from [28] and the student observations are adapted from [29].

the agent model consists of enforcing a distance-speed relationship between nodes and lane changing rules. These are discussed in the next two sections. In Section 4.4.3, the model is validated by comparing the size of platoons created by the model to those observed by Pushkarev and Zupan. As will be discussed in Section 5, with some small changes, the node interactions described here are also applicable to vehicles.

\subsubsection{Inter-node Distance-Speed Relationship}

When a node with a higher desired speed catches up to a slower moving node, it will either follow or pass. To understand the dynamics of catching up, it is necessary to understand the distance-speed relationship. The impact of this relationship is that nodes will be tightly packed (i.e. high density) if their speed is low (congestion), but if the speed is higher, then the nodes must be further apart (low density). Since the density of nodes plays an important role in the performance of mesh networks, the distance-speed relationship must be understood and realistically modeled. For both vehicles and pedestrians, these relationships have been extensively studied. Here we focus on the pedestrian case.

The distance-speed relationship for pedestrians was studied in [28] and [29]. Figure 8 shows the distance-speed relationship derived from these observations ${ }^{1}$. We approximate this relationship with $D(S)=S^{*} D_{\min } /\left(1.08 \times S^{*}-S\right)$ where $D_{\min }$ is the minimum distance between people without touching and $S^{*}$ is the desired speed of the pedestrian. $D_{\min }$ was found to be at least $0.35 \mathrm{~m}[12]$.

It has been found that pedestrian desired speeds are approximately Gaussian with mean 1.34 $\mathrm{m} / \mathrm{sec}$ and standard deviation $0.26 \mathrm{~m} / \mathrm{sec}[31,32,33]$.

\subsubsection{Lane changing}

While traffic lights are an important cause of platooning, the passing or not passing of slower walkers also plays an important role [12]. People will certainly not overtake slower walkers if

$\overline{1}$ The plot shown is based on area-speed relationships with the assumption of 0.75 meter of lateral space between people as found by Oeding [30]. 


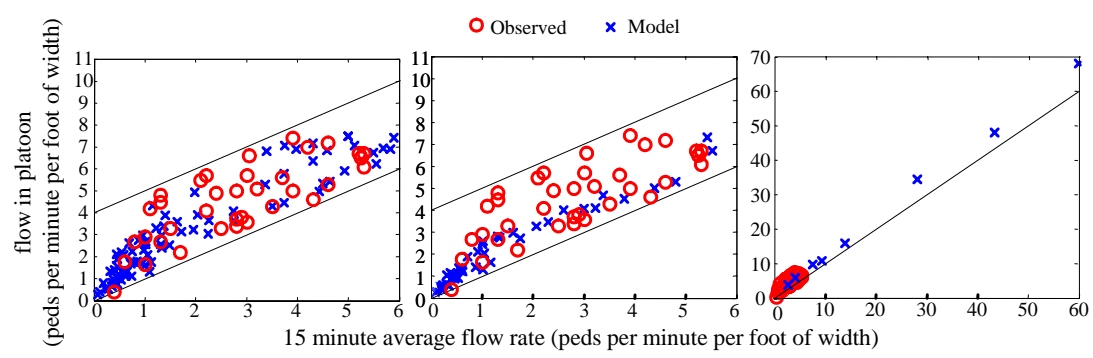

Fig. 9. A validation of the pedestrian agent model. The black lines are the ranges that Pushkarev and Zupan considered realistic. The circles are values that Pushkarev and Zupan observed and the $\mathrm{x}$-marks are the values generated by the simulator. The left-hand frame shows the results of the full simulator. The middle frame shows the results when no probabilistic passing model is used; instead a node always passes. The right-hand plot is when no inter-node dynamics are used, e.g., two nodes can occupy the same location.

there is no room (e.g., if the other lanes are full). Even if there is room, pedestrians (as well as vehicles) might not pass out of choice and select to slow down and follow the node ahead [34]. Such decisions lead to platooning.

While the dynamics of pedestrian overtaking has been observed, it has not been modeled. However, models for vehicle passing have been developed (e.g., [35]). We borrow from this model. It has been found that lane changing depends on the difference between the speed that results from not changing lanes and the speed that could be achieved if the lane was changed. Specifically, a slightly simplified model for the probability of wanting to change lanes and overtake a slower node is

$$
P(\text { desire to change lanes })=1 /\left(1+\exp \left(A+B\left(V_{*}-V^{*}\right)\right)\right)
$$

where $V_{*}$ is the speed that the node would achieve if it remains in the current lane and $V^{*}$ is the speed that would be achieved if the node changes lanes. Since speeds may experience short-term variation, instantaneous determinations of $V_{*}$ and $V^{*}$ leads to erratic behavior. Instead, letting $\nu$ denote the node that is considering changing lanes, we define $V_{*}$ to be the average speed of all nodes between $\nu$ and the next intersection, and define $V^{*}$ to be the minimum of the desired speed of $\nu$ and the average speed of the nodes in the target lane that would be between $\nu$ and the next intersection. Scaling the parameters found in [35], we set $A_{\text {Pedestrian }}=-0.225$, and $B_{\text {Pedestrian }}=1.7$.

While this model has not been verified for pedestrians, in the next section we will see that it does give rise to realistic platooning.

\subsubsection{Validation of the Agent model}

The burstiness of pedestrians has been investigated by Pushkarev and Zupan [12]. Their work has served as the basis for the pedestrian traffic engineering guidelines set forth in the Highway Capacity Manual [13]. The metrics of burstiness for pedestrian platoons is different from the ones typically used in studying data networks. Specifically, Pushkarev and Zupan 
compare two flow metrics, the 15 minute average flow rate (AFR) and the flow rate during a platoon (PFR). A node is in a platoon if the local density of nodes exceeds the average density. As is shown in Figure 9, the PFR is higher than the AFR. According to Pushkarev and Zupan, the larger the PFR is as compared to the AFR, the more bursty the pedestrian traffic. The study of Pushkarev and Zupan was not focused on finding the frequency of specific flow rates, but to examine what combinations of AFR and PFR occur on urban sidewalks. Thus, we use this data as a baseline with which we compare the pedestrian mobility model described above.

The left-hand plot in Figure 9 shows two sets of data. The generated data from the mobility model is from a variety of configurations including counting pedestrians on a block with and without buildings, various sizes of sidewalks (from 4 lanes to 32 lanes), various traffic light timings (from 60 seconds to 120 second periods), and various rates of pedestrians flowing into the street. As can be seen from the left-hand plot in Figure 9, the mobility model described above generates combinations of PFR and AFR that are realistic.

The center plot in Figure 9 shows the data set collected by Pushkarev and Zupan and a set of data generated by the mobility model but where nodes pass whenever there is room to pass, i.e., $P$ (desire to change lanes) $\equiv 1$ as oppose to what is given in (1). Clearly, increasing the propensity to change lanes acts to decrease the burstiness so that some realistic levels of burstiness never occur. Finally, the right-hand plot in Figure 9 shows Pushkarev and Zupan's data compare to data generated by the mobility model but where there are no inter-pedestrian dynamics, i.e., nodes move along lanes irrespective of other nodes. Such mobility allows, for example, nodes to disobey the distance-speed relationship. As shown in Figure 9, ignoring inter-node dynamics results in unrealistic levels of congestion (extreme discomfort occurs when the flow rate exceeds $7[12])$.

\subsection{Mode of Travel During Commute}

People may travel to and from work by car, by subway, and, for people who live within the simulated area, by walking. In the case of traveling to work by car, the trajectory of the person and the car matches until the car arrives at a parking-lot to which the person is assigned (see Section 4.1). Once the person reaches the parking-lot, they walk to their destination. Street parking is not considered here, but is considered by other traffic micro-simulators (e.g., [36]).

During subway travel, the person's trajectory starts at the subway stop and the person walks from the subway to their destination. It is assumed that subway trains arrive at Poisson distributed times, and hence people exit the subway in bursts. As mentioned in [12], subway train arrivals can lead to platooning or clusters of pedestrians. Realistic mean times between subway arrivals is $3-10$ minutes [37].

In American cities, the fraction of people who take mass transit widely varies, hence the UDel Models simulator allows this fraction to be adjusted. As points of reference, the national average of people who take mass transit to work in the US is 10.4 [38], but $87 \%$ of the people 
who enter Manhattan use mass transit [39].

\subsection{Urban Population Size}

It is well known that the number of users has a major impact on the performance of the network. Thus, realistic node population size is an important part of realistic simulation. While the number of nodes in a network depends on the number of people in the simulated region, it also depends on the fraction of people that subscribe to the network. Today, mobile phone penetration in Europe exceeds $80 \%$, while in the US the fraction of subscribers is approximately $60 \%$. Of course, in the early period of mobile phone deployment, the fraction of subscribers was much smaller. Hence, a wide range of penetration rates are realistic.

As expected, realistic populations size in an urban region can be quite large. For example, $1 \mathrm{~km}^{2}$ of Manhattan may contain 10,000 people outdoors [12], a number that is far larger than most simulations currently found in the literature. However, if $10 \%$ of the population participates in the network, then a nine block region of Chicago would contain about 4000 nodes, a number that can be supported by protocol simulators such as QualNet [40]. The following presents guidelines for determining the population size in an urban region.

In the urban core, most of the indoor space is used for commercial purposes, including offices, stores, and restaurants, with office space being the most prevalent. As one moves away from the core, a larger fraction of the indoor space is used for residences. However, it is assumed that the map specifies which buildings contain offices, stores, residences, or a mixture. For office space, a survey of office use in the UK found that typical densities are approximately $15 \mathrm{~m}^{2}$ per person [41]. Thus, the total working population can be determined from the total area of office space.

The US Census American Housing Survey finds that in urban areas there is approximately 1 person per $65 \mathrm{~m}^{2}$ of residential space. Thus, the size of the residential population can be computed from the total area of residential space. However, in the UDel Models it is assumed that $92 \%$ of the people that live in the city will also work within the city, and hence are counted in the working population (the other $8 \%$ are not working).

The UDel Models sets the population as follows 
Number of office workers $=\frac{\text { Total office area }}{15}$,

Number of people living within the city

$$
=\min \left(\frac{\text { Total residential area }}{65}, \frac{\text { Number of office workers }}{0.92}\right) \text {, }
$$

Number of people simulated $=$ Number of office workers

+ Number of people living within the city $\times 0.08+$ Number of nonworking visitors,

Number of people who commute via subway $=$ MassTransitRatio $\times$

(Number of office workers - Number of people living within the city $\times 0.92$ ),

Number of people who commute via car $=(1$-MassTransitRatio $) \times$

(Number of office workers - Number of people living locally $\times 0.92$ )

where the values are such that the office worker density is maintained even if there is an abundance of residential space. Note that we allow for some nonworking visitors. These people follow the same mobility as nonworkers that live within the city. However, further work is required to determine realistic sizes of the nonworking visitor populations. The MassTransitRatio is the fraction of commuters that take the subway, as discussed in Section 4.5 .

Remark 1 Some population density statistics focus only on the number of residences per geographic area, not per square foot of indoor space. Furthermore, in the urban core, workers will commute to work, and hence are not counted in the residence population densities that are commonly cited.

\section{$5 \quad$ Vehicle Mobility}

Vehicle mobility has been widely studied within urban planning and sophisticated simulators exist (e.g., [42]). However, these simulators focus on highway traffic and often require more detailed information than is easily accessible to network researchers. On the other hand, simulation of vehicles along urban streets is more simple than simulation of highways where complicate processes such as rubbernecking can have a dramatic impact on traffic.

In general there are two types of vehicles, namely, commercial vehicles such as delivery vehicles and busses that make frequent stops, and private vehicles that make few stops. The UDel Models only considers private vehicles. For private vehicles, two types of trips are considered, trips where the car simply passes through the simulated region, and trips where the vehicle carries a person into or out of the simulated region. We first examine the case when the car simply passes through the simulated region.

Like the pedestrian model, a hierarchical model is used. However, only two tiers are used. The highest tier controls the flow of vehicles into the simulated region, while the lower tier controls the mobility of the vehicles. The lower tier is discussed next. 

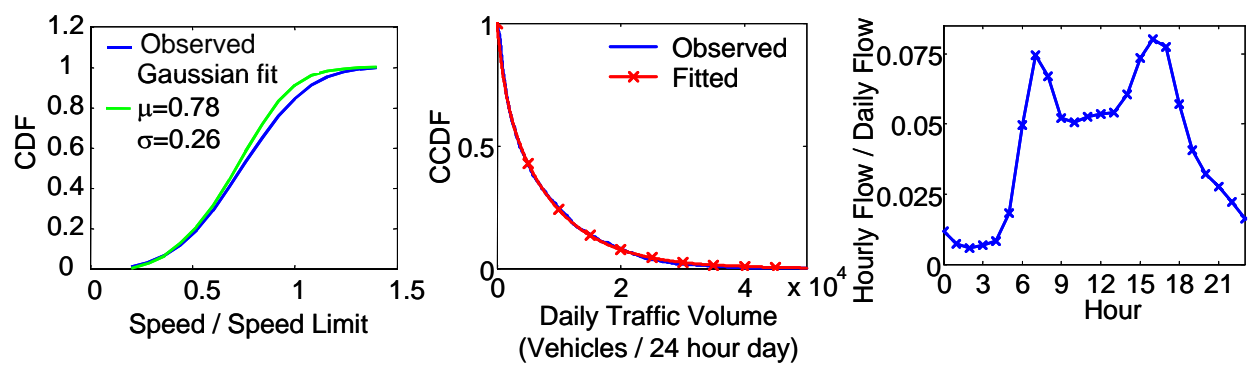

Fig. 10. Left: The cumulative distribution Function (CDF) of the vehicle speed in urban areas and a fitted Gaussian CDF. Middle: The complementary cumulative distribution function of the vehicle traffic volumes on San Francisco streets. Right: The ratio of hourly voume to daily volume.

\subsection{Vehicle Agent Model}

This lower tier is similar to the pedestrian mobility in that it includes the same structure for node interactions; specifically, the same framework for passing and speed-distance relationship is used. The distance-speed relationship is given by $D(S)=\alpha+\beta S$. For dry driving conditions, it has been found that $(\alpha, \beta)$ ranges from $(1.78,10.0)$ to $(1.45,7.8)[43]$. These values also agree with the observations presented in [44] and [45]. The probabilistic passing model is discussed in Section 4.4.2, but the parameters in (1) are $A_{\text {Vehicle }}=-0.225$, $B_{\text {Vehicle }}=0.1$.

For vehicles, the ratio of the vehicle's desired speed to the speed limit presented in [46] can be modeled as Gaussian with mean 0.78 and standard deviation 0.26 (see Figure 10).

Traffic engineering provides guidance on modeling the paths cars take through the modeled area. Traffic simulators / congestion prediction models such as CORSIM [42] allow vehicle trips to be generated in two ways, with origin-destination (O-D) flow matrices or with turning probabilities. O-D matrices are much like the traffic matrix used in data network provisioning. The rate at which vehicles enter the simulated region at a origin $\mathrm{O}$ with desired destination $\mathrm{D}$ is given by the $(\mathrm{O}, \mathrm{D})$ element of the O-D matrix. If only turning probabilities are used, vehicles enter into the modeled area at one of a pre-selected locations and proceed until the vehicle arrives at any exit location, which is at the edge of the modeled area or a parking location. At each intersection, vehicles turn or go straight according to the turning probabilities assigned to that intersection. O-D matrices yield a more accurate simulation, however, accurate O-D matrices are difficult to determine, whereas turning probabilities can be determined by simply counting vehicles turning at each intersection. Thus, both approaches are used for urban traffic engineering.

Drawbacks of turning probabilities are that vehicles might travel in long loops or meander through the city of extended periods for time. However, since cars typically go straight ( turning probabilities are typically between 0.1 and 0.3 [47], [48]) such unrealistic behavior is rare; most trips proceed through the city with only a few turns. The UDel Models currently uses homogeneous turning probabilities. 


\subsection{Vehicle Flow Rates}

Each road that reaches the edge of the simulated area may have vehicles enter or exit at that point. Following the findings of [49], it can be assumed that vehicles enter the region as if they have just passed through a traffic light (i.e., in bursts), and that the number of vehicles in a burst is distributed according to a Poisson distribution. The mean number of vehicles per burst is not the same for each road. The distribution of flow rates for San Francisco streets is shown in middle frame of Figure 10 [50]. As is also shown, this distribution is well modeled by the mixture of two exponentials, specifically, $P$ (Number of cars per day $>r$ ) $=$ $0.74 \exp \left(-r / 8.9 \cdot 10^{3}\right)+(1-0.74) \exp \left(-r / 1.3 \cdot 10^{3}\right)$. To convert the daily average flow shown in Figure 10 to hourly flow, the scale factor from [51] is shown in the right-hand frame of Figure 10 is used. Note that a simple way to scale the amount of traffic that enters a city is to scale the distribution of daily volume, but leave the scale factor unchanged to get realistic variability of traffic flow.

While many vehicles may pass through the city, they may also carry people into or out of the city (we ignore the possibility that people use a car to travel within the city). In the UDel Models, when a person desires to exit the city via a car, they merely walk to their parking-lot. Upon reaching the parking lot, the person enters a car and then proceeds to drive through the city until exiting the city. Similarly, when a person desires to enter the city, the next unoccupied car that enters the city is assigned to the person. This car proceeds to the desired parking-lot assigned to the person. Upon arriving, the person exits the vehicle and walks to their office. While driving, the trajectory of the car is the same as the trajectory of the person. Furthermore, depending on the transmitter and propagation, communication with the person may be possible. However, when a person or a car are not in the simulated area, they are no longer reachable as all channel gains are set at $-\infty \mathrm{dB}$.

\section{Impact of Mobility on Network Performance}

This section investigates the impact of realistic mobility on simulated network performance. A detailed investigation of network performance and mobility models is difficult since the impact on the network performance depends on the metric and/or protocol(s) of interest. Furthermore, there is a large number of types of mobility (e.g., indoor, outdoor, pedestrian, and vehicle), and, as described above, there are many aspects of mobility (e.g., speed and node interaction). Thus, only of some of the impacts that realistic mobility has on performance are examined.

In the analysis that follows, the realistic mobility is generated by the UDel Models version 2.0 [15]. The simulations are based on a $3 \times 3$ block region of downtown Chicago with 54 fixed wireless relays placed on lampposts that are uniformly distributed throughout the region. The UDel Models was also used to generate realistic propagation for this region [11]. In several experiments below, CBR traffic is sent from a base station to a mobile node, where 


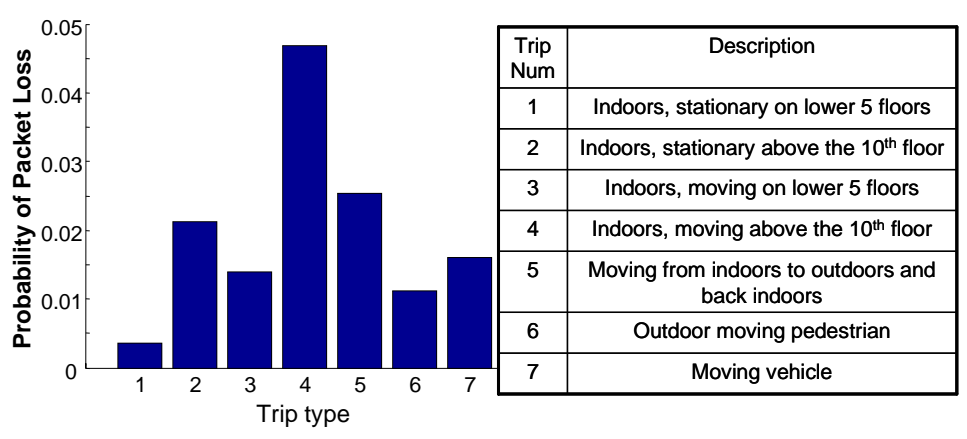

Fig. 11. Loss probability for different types of trips.

the base station was located in the north west corner of the simulated region. The CBR traffic consisted of 100B packets every 500 msec. $802.11 \mathrm{~b}$ at $2 \mathrm{Mbps}$ was used for all transmissions with RTS/CTS enabled. AODV routing was used [52].

\subsection{Trip Types}

Urban mobility includes a diverse set of types of trips. In order to explore the impact of the trip type on network performance, seven types of trips were defined (see Figure 11). For each trip type, a mobility trace for the time period 2:45 p.m. to 3:15 p.m. was searched for the desired type of trip. An application configuration file was generated so that CBR traffic was sent from the base station to the mobile node during the desired trip. There was only one flow for each simulation trial. The simulations each ran for 100 seconds, except, for indoors to outdoors to indoors trips, the simulation started as the node began to move and ended when it stopped moving. It was ensured that such trips lasted at least 100 sec. Figure 11 shows the loss probability for the different trip types averaged over 10 trials. Clearly, the trip type has a significant impact on the performance. While the quantitative impact of the trip type could not be predicted, as explained next, the qualitative impact is expected.

Since nodes on the lower floors are within the communication range of the infrastructure nodes, routes from the base station to nodes on the lower floors are likely to be composed of infrastructure nodes. Consequently, connections to stationary nodes on the lower floors (Trip Type 1) have low loss probability. Due to paths failures due to the mobility, connections to nodes moving on the lower floors (Trip Type 3) suffer more losses than stationary nodes on the lower floors. However, since these nodes remain within the communication range of the infrastructure, the loss probability remains low. In contrast, paths to stationary nodes on the upper floors must include mobile nodes on other floors. Hence, connections to stationary nodes on upper floors (Trip Type 2) suffer more losses than connections to nodes on the lower floors. And when the nodes on the upper floors move, the loss probability is further increased.

Wireless signals propagate much further outdoors than indoors. Thus, connections to outdoor pedestrians (Trip Type 6) have few path failures and hence experience low loss probability. Due to the higher rate of mobility, connections to vehicles (Trip Type 7) have a slightly 
higher loss probability. Finally, connections to nodes that move from indoors to outdoors and back indoors (Trip Type 5) experience a fairly high loss probability. This behavior is due to the path failures that occur when the node moves from indoors to outdoors and back indoors and when the node is changing floors. Note, that Trip Type 4 does not distinguish between whether the node's starting or ending point was on the upper or lower floors.

\subsection{Random Office Waypoint}

Random waypoint is a popular mobility model for exploring the performance of MANETs. Random office waypoint (ROW) is an urbanized extension of random waypoint. In this model, a pedestrian walks to a randomly selected office. Upon reaching the office, the pedestrian pauses for an exponentially distributed amount of time, and then selects a new office and repeats the process. The office pause times are set to 19 minutes, matching the office pause times of the UDel Mobility Model. In the next three subsections, the ROW model is compared to realistic mobility.

\subsubsection{Trip Types}

In order to compare the impact that the ROW model and realistic mobility model have on performance, the destination of a test connection was selected in three ways. First, the destination was selected so that when the simulation began, the node was stationary and indoors. Second, the destination was selected so that when the simulation began, the mobile node had just begun to move. Third, the destination was selected at random regardless of whether the node is moving. In all cases, the simulation ran for 300 sec. Section 6.1 showed that the floor that a node is on has a significant impact on the packet loss probability. Thus, in order to focus on the impact of mobility and not be distracted by the floor that nodes are on, in this section, all nodes were restricted to the lower five floors of buildings.

The main difference between ROW and realistic mobility is that in ROW, nodes tend to take long outdoor trips, whereas in realistic urban mobility, most mobile trips are short and do not include an outdoor component. As a result, in ROW, nodes tend to spend more time moving and much of this time is outdoors. As explained next, the impact of these differences are detectable in packet loss probability shown in Figure 12.

Since nodes often take long outdoor trips in the ROW model, there are more mobile nodes outdoors under the ROW model than under realistic mobility. Consequently, under the ROW model, a route found by AODV from an outdoor base station is more likely to include outdoor mobile nodes than it is under realistic mobility, in which case the route is more likely to use the lamppost mounted relays. As a result, connections to stationary nodes have a higher loss probability under the ROW model than under realistic mobility. In the mobile case, under the ROW model, nodes tend to take indoor-outdoor-indoor trips (since a randomly selected next office is typically in a different building). As shown in Figure 11, indoor-outdoor-indoor trips suffer a higher loss probability than indoor trips, which dominate realistic mobility. 


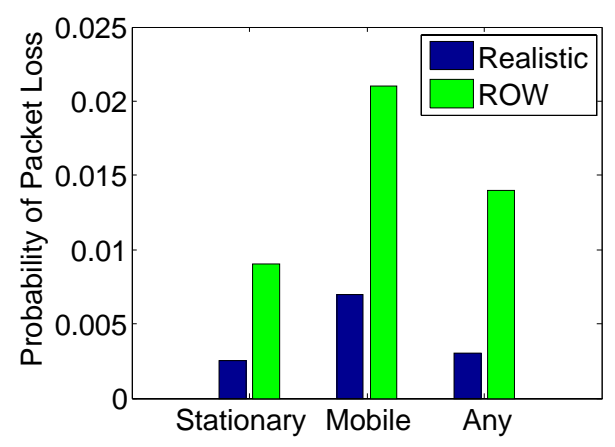

Fig. 12. Packet loss probability for random office waypoint (ROW) and realistic mobility for different types of trips.

Finally, under realistic mobility, nodes take short trips, and hence spend much of their time not moving. Thus, when a node is selected at random, the probability of packet loss for realistic mobility is similar to the probability of packet loss of stationary nodes. On the other hand, under the ROW model, nodes spend a larger fraction of time moving. Thus, when a node is selected at random, the probability of packet loss is approximately half way between the value obtained when the nodes are stationary and the value obtained when the nodes are mobile.

\subsubsection{Node Clustering}

As mentioned in Section 4.4, node interactions lead to node clustering. Thus, one result of node interactions is that nodes will have a larger number of nearby neighbors than if nodes are more uniformly spread. Figure 13 demonstrates this effect by showing the average number of neighbors as a function of the channel loss. Specifically, let $N_{i}(H)$ be the number of nodes $j$ such that the channel loss between node $i$ and $j$ is at least as strong as $H$. The left-hand side of Figure 13 shows the average value of $N_{i}(H)$, averaged over all outdoor nodes $i$. This figure shows the average when node interaction is enabled and when it is disabled. The right-hand side of Figure 13 shows the ratio of the average value of $N_{i}(H)$ when node interaction is enabled and the average value of $N_{i}(H)$ when node interaction is disabled. Since the channel to nearby nodes have low channel loss, clustering results in an increase in the number of nodes with low channel loss. For example, the average number of nodes with a channel loss lower than $10 \mathrm{~dB}$ is increased by nearly a factor of two. However, clustering does not greatly impact the number of nodes that are at greater distances (e.g., with channel loss of $60 \mathrm{~dB}$ ). Of course, the impact that clustering has on network performance depends on the metric and protocol. For example, clustering will increase interference with nearby nodes. However, with such good channels, very high data rate communication to nearby nodes is possible. This property could be used to construct ad hoc virtual antennas.

\subsubsection{Mobility Management - The Number of Infrastructure Nodes Seen}

Large-scale mesh networks are expected to have thousands of mobile users. Advanced mobility management schemes are necessary to support user mobility in a scalable fashion. In 

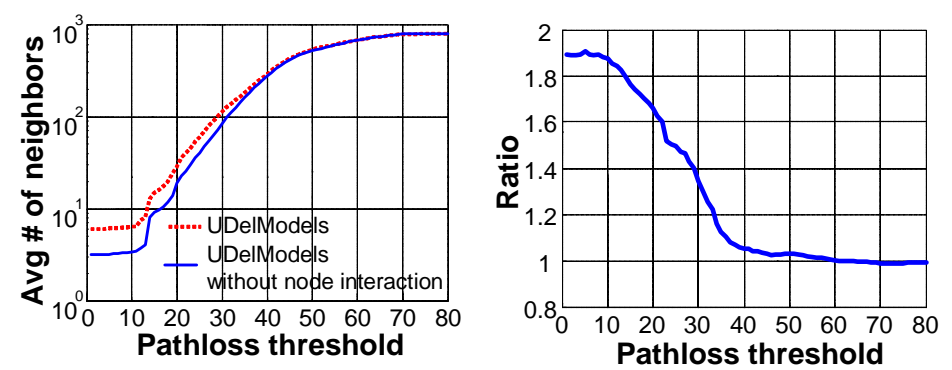

Fig. 13. Impact of clustering. Clustering results in nodes having a larger number of neighbors with low channel loss than if the nodes are not clustered. As shown, clustering increases number of neighbors with low channel loss.

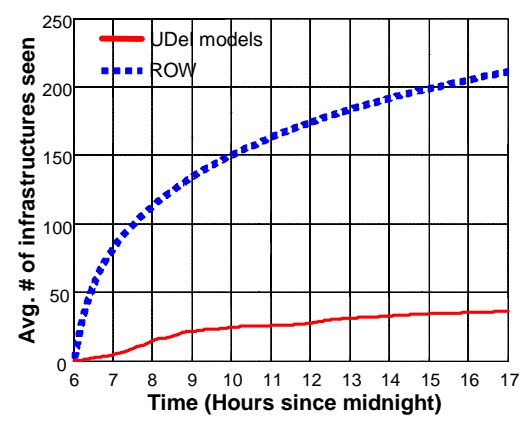

Fig. 14. Average cumulative number of infrastructure nodes heard by a mobile node throughout the day for the Random Office Waypoint (ROW) mobility model and the UDelModels mobility model.

order to determine the performance of such mobility management schemes, realistic mobility is required. For example, if users are only able to communicate with a small number of infrastructure nodes (INs) through the course of a typical day, it is feasible for the INs to maintain per user profiles. However, such an approach would not be feasible if users typically visit a large number of INs. Figure 14 shows the average of the cumulative number of INs that a user hears throughout the day for UDel Models and the ROW model. In the case of the UDel Models, the beginning of the day is marked by a rapid increase in the cumulative number of infrastructure nodes heard. However, once most people arrive at work (around 9 a.m.), the rate that new INs are heard decreases. Since people may explore new areas of the city during a lunch time trip, the number of new INs heard slightly increases around noon. On the other hand, under the ROW model, nodes continually explore large regions of the city. Hence, a large number of INs are heard and the number heard continually increasing throughout the day. Thus, maintaining per user profiles appears considerable more difficult under the ROW model than under realistic mobility.

\subsection{Node Interaction in Vehicle Networks}

The dynamics of car mobility includes passing, queuing at traffic lights, and obeying traffic lights. Without these dynamics, cars move at a constant speed while occasionally making turns. Such a model is similar to the Manhattan Mobility Model [53]. In order to investigate the impact of the vehicle mobility model, the CBR data traffic was sent from a base station to 
a randomly selected car. The cars were selected at random under the constraint that the car remained in the simulated region for at least 100 seconds, which was the connection duration. The simulated time was 5:30 p.m. It was found that the full mobility model resulted in a loss probability of 0.016 , whereas when the dynamics of vehicle mobility was neglected, the loss probability jumped to 0.064 , an increase by a factor of four. Recall that TCP provides very low throughput when the loss probability exceeds $5 \%$ [54]. Thus, the realistic mobility results in the conclusion that TCP will perform reasonably well, whereas, the unrealistic model leads to the opposite conclusion.

\section{Related work}

There are several mobility models used for MANET simulation. The most popular is the random way-point model [55]. There are many variations of such random mobility models (see [56] for details and references). However, these models are obviously not realistic. In [57], several scenario based mobility models were considered. However, as mentioned in [57], these mobility models are not meant to be realistic. In [53], the Manhattan mobility model is introduced where nodes are restricted to a grid that resembles the street map of Manhattan. This model does not include any realistic node mobility dynamics (e.g., node interaction, traffic lights) or realistic trip generation. While Manhattan used idealized grid-cities, several researchers have used actual city maps from the TIGER data sets [19] (e.g., [20, 22, 21]), whereas [58] uses a random graph. In many of these graph constrained cases, the mobility is essentially random way-point, but restricted to a graph. In [59], mobility patterns from multi-user games were used, but did not verify that the mobility of characters in games to resemble the mobility of pedestrians or vehicles.

In $[57,16,60]$ obstacles were included, and mobile nodes avoid the obstacles. In [16] and [60], the obstacles were randomly located buildings. As was mentioned in Section 3, as well as in [17], streets play an important role in connectivity. Hence, the random placement of buildings will result in non-realistic topologies.

Recently there has been interest in developing more detailed models along the lines discussed here. For example, [61] and [62] discuss an empirical model based on observations of pedestrians on a university campus, while [63] describes a realistic model of communication usage during disasters.

One of the most detailed mobility models is GEMM [64]. GEMM is an agent-based model where several factors impact the mobility of the node. For example, GEMM includes attraction points as well as habits to influence the mobility. A noted drawback of this work is that realistic values of the model parameters are not known.

Metropolitan Adhoc Network Simulator (Madhoc) is a scenario-based simulator that allows simulation of several different urban scenarios. The Madhoc simulator is currently under development [65]. 
In [66], a type of trace-based urban mobility is presented. This algorithm takes as input the flow edge rates of a simulated area, i.e., the rate that pedestrians enter or exit the simulated region at each walkway that crosses the edge of the simulated region. From these values, mobility within the simulated region is estimated. Since this scheme uses actually mobility measurement, it is inherently realistic. However, it is difficult to extend the data to other scenarios for which data was not collected. Furthermore, the approach is best suited for mobility in a small region, e.g., mobility within a large urban mass transportation station.

In [67], node mobility is based on a predefined social network; nodes tend to move towards nodes with which they have a strong social connection. Such a mobility model could be incorporated into the task model in Section 4.3 so that the nodes that take part in meetings are those that have strong connections in a social network. This approach could also be used in group mobility.

In [22], a realistic mobility model of vehicles is developed. While the model does not include realistic flow rates, it does include a realistic distance-speed relationship as well as a realistic desired speed distribution (i.e., Gaussian, as is discussed in Section 5). The model also makes use of realistic maps via TIGER [19]. However, a probabilistic passing model such as the one described in Section 4.4.2 is not included.

While realistic mobility within mobile wireless networking is a new area of interest, within disciplines such as urban planning, architecture, transportation engineering, and sociology, mobility modeling is a mature field with early efforts dating back nearly fifty years [68]. While these areas have produced refined techniques, the objectives of this previous work are different from what is required for modeling mobile wireless networks. Thus, it is not possible to simply copy these other efforts. Rather, techniques, observations, and results have been taken from this large body of work and adapted to the specific needs of mobile wireless networks. While it is not possible to provide a complete review of these active research areas, a brief overview is as follows.

Much of this previous work in mobility can be classified into flow based [69, 70, 71, 72, 73] mesoscopic [74, 75], cellular automata [76, 77, 78] agent-based [79, 80,81, 82, 83, 84] or activity-based [85, 86, 87] methods. Flow-based methods do not model individual mobile nodes, but model the density of nodes in continuous flows. Mesoscopic models aggregate nodes into groups. Cellular automata discretize space and model the node density in each cell. Since mobility modeling requires each node to be modeled individually, these three methods are not appropriate for networking. Thus, the methodology presented above incorporates the activity-based and agent-based approaches.

The data for vehicle agents is spread throughout the literature $[47,88,89,90,91,92,75]$. For pedestrian mobility, observations can also be found in the literature (e.g., [93, 94, 72, 95, 96, 87, 97]), but Pushkarev and Zupan [12] and Fruin [98] have compiled a collection of their own observations as well as other researchers' observations. Their work is the authoritative work on pedestrian mobility and forms the basis for the pedestrian mobility in the US Highway Capacity Manual [99]. 
CORSIM (corridor simulator) [42] is by far the most widely used traffic simulator for highway and traffic planning [100]. CORSIM is used for accurate traffic prediction. As a result, CORSIM is more realistic than the vehicle mobility discussed here, but it is also far more difficult to configure and use.

\section{Future Work in Mobility}

There are several areas of realistic urban mobility simulation that require further effort. One important area is mobility during disasters, crisis, and other events (e.g., Independence Day celebrations). Disasters and crisis mobility requires mobility models not only of the civilians, but also of emergency personnel. Note that both Philadelphia and San Francisco specify that their mesh network will be used to enhance emergency communication. Also, the discussion above and the current version of the UDel Models only considers cars. However, buses and commercial trucks should also be considered. For example, network protocols for such commercial vehicles are already under development (e.g., [101, 102, 103]).

The mobility models develop above are mostly derived from statistics collected in the US. However, use of time and the agent models of both vehicles and people depend on the country. Much of the data used here is also available for other countries. Hence, future work will develop mobility models for cities in other countries besides the US. Similarly, the focus of the model is mostly on office workers and nonworkers, the dynamics of nonoffice workers still needs to be explored and incorporated into the simulator.

Group mobility is a popular class of mobility models. However, there has been little work on realistic group mobility. One situation where group mobility commonly occurs in the urban setting is when groups of office workers go to lunch. An informal study performed in Philadelphia found that the number of people in a group followed the Zipf distribution with shape parameter of 2.18 , i.e., $P$ (Group size $\geq g)=1 / g^{2.18}$. However, further study of group sizes and group mobility dynamics is required.

\section{Conclusions}

A methodology for realistic simulation of urban mobility was presented. The techniques described have been implemented in a suite of simulation tools that are available for download [15]. The techniques presented are based on data collected from a wide range of sources. For example, the activities that people perform are derived from the 2004 US Bureau of Labor Statistics survey on time use. The detailed mobility model of people and vehicles is derived from modeling methodologies and data collected by the urban and traffic planning community. Vehicle traffic flows are derived from data collected by the City of San Francisco and the State of Connecticut. The density of people are derived from surveys of office space use and the US Census American Housing Survey. Other aspects of the model are derived 
from other data. In all, much of the model is based on observations of the mobility of people and vehicles.

While the mobility model presented here is considerably more realistic than models often used in mobility wireless networking research, realistic mobility alone will not produce realistic simulations. Along with realistic protocol and physical layer simulation, it is critical to model the channel realistically. Therefore, the techniques discussed here are incorporated into a simulation package such as [15] that includes propagation simulation.

\section{References}

[1] K. Dell, Welcom ro wi-fi-ville, Time Magazine.

[2] The City of Corpus Christi, Corpus Christi WiFi (2007).

[3] Philadelphia Mayor's Office, Government leaders teleconference - wireless: The 21stCentury techhology (2004).

URL http://www.phila.gov/mois/press/multimedia/pdfs/Wireless Philadelphia092004.pdf

[4] Unstrung, SF, EarthLink agree on muni net, available at http://www.unstrung.com/document.asp?doc ${ }_{i} d=113945 W T . s v l=n e w s 1_{1}$.

[5] H. Xie, S. Tabbane, D. Goodman, Dynamic Location Area Management and Performance Analysis, in: Proceedings of 43rd IEEE Vehicular Technology Conference, 1993, pp. 535-539.

[6] J. Markoulidakis, G. Lyberopoulos, D. Tsirkas, E. Sykas, Evaluation of Location Area Planning Scenarios in Future Mobile Telecommunication Systems, in: Wireless Networks, Vol. 1, 1995, pp. 17-29.

[7] S. J. Kim, C. Y. Lee, Modeling and Analysis of the Dynamic Location Registration and Paging in Microcellular Systems, in: IEEE Transactions on Vehicular Technology, Vol. 45, 1996, pp. 82-90.

[8] A. Hac, Z. Zhou, Locating Strategies for Personal Communication Networks: A Novel Tracking Strategy, in: IEEE Journal on Selected Areas in Communication, Vol. 15, 1997, pp. 1425-36.

[9] A. Bar-Noy, I. Kessler, Tracking Mobile Users in Wireless Networks, in: IEEE Transactions on Information Theory, Vol. 39, 1993, pp. 1877-1886.

[10] S. Bohacek, V. Sridhara, L. Lou, Efficient paging in large-scale urban mesh networks, in: Proceedings of the 4th International Workshop on Wireless Mobile Applications and Services on WLAN Hotspots, No. Available at http://udelmodels.eecis.udel.edu, ACM, 2006, pp. $81-90$.

[11] V. Sridhara, S. Bohacek, Realistic propagation simulation of urban mesh networks, ComnetsHttp://udelmodels.eecis.udel.edu.

[12] B. Pushkarev, J. M. Zupan, Urban Space for Pedestrians, MIT press, 1975.

[13] Transportation Research Board, 2000 Highway Capacity Manual, National Research Council, Washington, D.C., 2000.

[14] A. Szalai, The Use of Time, mouton, the hague, 1972. 
[15] S. Bohacek, V. Sridhara, J. Kim, UDel models for simulating urban wireless networks, available at http://UDelModels.eecis.udel.edu/.

[16] A. Jardosh, E. M. Belding-Royer, K. C. Almeroth, S. Suri, Towards realistic mobility models for mobile ad hoc networks, in: MobiCom, 2003.

[17] S. Bohacek, V. Sridhara, The graphical properties of MANETs in urban environments, in: The Forty-Second Annual Allerton Conference on Communication, Control, and Computing, 2004.

[18] Geographic information system (GIS), http: www.gis.com.

[19] U. C. Bureau, Topologically integrated geographic encoding and referencing (TIGER), Available at http://www.census.gov/geo/www/tiger/.

[20] R. Mangharam, D. S. Weller, D. D. Stancil, R. Rajkumar, J. S. Parikh, GrooveSim: A topography-accurate simulator for geographic routing in vehicular networks, in: Proceedings of the 2nd ACM International Workshop on Vehicular Ad Hoc Networks, ACM, Cologne, Germany, 2005, pp. 59-68.

[21] A. K. Saha, D. B. Johnson, Modeling mobility for vehicular ad-hoc networks, in: Proceedings of the 1st ACM International Workshop on Vehicular Ad Hoc Networks, ACM, Philadelphia, PA, 2004, pp. 91-92.

[22] D. R. Choffnes, F. E. Bustamante, An integrated mobility and traffic model for vehicular wireless networks, in: Proceedings of the 2nd ACM International Workshop on Vehicular Ad Hoc Networks, ACM, Cologne, Germany, 2005, pp. 69-78.

[23] N. C. Romano, J. F. Numamaker, Meeting analysis: Findings from research and practice, in: Proceedings of Teh 34th Hawaii International Conference on Systems Science, 2001.

[24] U. D. of Labor Bureau of Labor Statistics, American time use survey (ATUS), http://www.bls.gov/tus/ (2003).

[25] V. Sridhara, J. Kim, S. Bohacek, Performance of urban mesh networks, in: The 8Th ACM/IEEE International Symposium on Modeling, Analysis and Simulation of Wireless and Mobile Systems, 2005.

[26] R. R. Panko, S. T. Kinney, Meeting profiles: Size, duration, and location, in: Proceedings of The 28th Annual Hawaii International Conference on Systems Science, 1995.

[27] R. K. Mosvick, R. B. Nelson, We've Got to Start Meetings Like This!, Scott, Foresman and company, Glenview, Illinois, 1987.

[28] S. J. Older, Movement of pedestrian on footways in shopping street, traffic engineering and control (1968) 160-163.

[29] F. P. D. Navin, R. J. Wheeler, Pedestrian flow characteristics, traffic engineering (1969) 30-36.

[30] D. Oeding, Traffic loads and dimensions of walkways and other pedestrian circulation facilities, Strassenbau and strassenverkehrstechnik 22.

[31] D. Helbing, Sexual differences in human crowd motion, Nature 240 (1972) 252.

[32] D. Helbing, The statistics of crowd fluids, Nature 229 (1971) 381.

[33] G. K. Still, Crowd dynamics, Ph.D. thesis, university of warwick (2000).

[34] Y. Zhang, L. E. Owen, J. E. Clark, A multi-regime approach for microscopic traffic simulation, in: The 77th TRB Annual Meeting, 1998.

[35] K. I. Ahmed, Modeling drivers' acceleration and lane changing behavior, Ph.D. thesis, 
MIT (1999).

[36] V. Mauro, Evaluation of dynamic network control: Simulation results using NEMIS urban micro-simulator, in: Transportation Research Board Annual Meeting, Washington D.C., 1991.

[37] M. B. T. Authority, Available at http://www.mbta.com.

[38] Http://www.transact.org/report.asp?id=190.

[39] Http://www.transalt.org/info/SOVBanImpacts.pdf.

[40] Scalable Network Technologies, The QualNet simulator http://www.qualnet.com/.

[41] M. Wist, Office space - how much is enough?, Tech. rep., Gerald Eve (2001).

[42] FHWA, CORSIM UserŠs Manual, Version 5.0, ITS Research Division, FHWA (2000).

[43] S. Shekleton, A GPS study of car following theory, in: Conference of Australian Institutes of Transport Research (CAITR), 2002.

[44] T. Dijker, P. H. L. Bovy, R. G. M. M. Vermijs, Car following behavior in different flow regimes, in: P. H. L. Bovy (Ed.), Motorway Traffic Flow Analysis, delft university press, Delft, the Netherlands, 1998, pp. 49-70.

[45] J. Piao, M. McDonald Analysis of stop and go driving behavior through a floating vehicle approach, in: Proc. Of the IEEE Inteligent Vehicles Symposium, 2003.

[46] J. Du, L. Aultman-Hall, An investigation of the distribution of driving speeds using invehicle GPS data, in: Vermont Institute of Transportation Engineers Annual Meeting, available at http://www.neite.org/vt/dist $1_{2} 004 /, 2004$.

[47] J. E. Hummer, Unconventional left-turn alternatives for urban and suburban arterials, ITE Journal 68.

[48] M. J. Bayarri, J. O. Berger, G. Molina, N. M. Rouphail, J. Sacks, Assessing uncertainties in traffic simulation: A key component in model calibration and validation, Tech. Rep. 137, National Institute of Statistical Sciences (2003).

[49] A. Kamarajugadda, B. Park, Stochastic traffic signal timing optimization, Tech. Rep. UVACTS-15-0-44, Center for transportation studies at the university of Virginia (2003).

[50] City of San Francisco Department of Parking and Traffic, Daily traffic volume, Available at http://www.sfgov.org/site/ uploadedfiles/dpt/Volumes

[51] J. N. I. A. W. M. E. Dessouki, M. Zhao, F. Guo, Estimating link traffic volumes by month, day of the week, and time of day, Tech. Rep. JHR 02-287, Joint Highway Research Advisory Council of the University of Connecticut (2002).

[52] C. E. Perkins, E. M. Royer, Ad hoc on-demand distance vector routing, Proceedings of the 2ns IEEE Workshop on Mobile Computing Systems and Applications (1999) $90-100$.

[53] F. Bai, N. Sadagopan, A. Helmy, Important: a framework to systematically analyze the impact of mobility on performance of routing protocols for adhoc networks, in: InfoCom, 2003.

[54] S. Bohacek, K. Shah, A model of TCP's throughput and time-out probablity - steadystate and and time-varying dynamics, in: GlobeCom 2004.

[55] D. Johnson, D. Maltz, Dynamic source routing in ad hoc wireless networks, in: Imielinski, Korth (Eds.), Mobile Computing, kluwer academic publishers, 1996, pp. 153-181.

[56] T. Camp, J. Boleng, V. Davies, A survey of mobility models for ad hoc network research, WCMC 2 (5) (2002) 483-502. 
[57] P. Johansson, T. Larsson, N. Hedman, B. Mielczarek, M. Degermark, Scenario-based performance analysis of routing protocols for mobile ad-hoc networks, in: MobiCom, 1999.

[58] J. Tian, J. Hahner, C. Becker, I. Stepanov, K. Rothermel, Graph-based mobility model for mobile ad hoc network simulation, in: Proceedings of the 35th Annual Simulation Symposium, 2002, pp. 337-345.

[59] F. H. P., Fitzek, L. Badia, P. Seeling, J. G. Schulte, T. Henderson, Mobility and stability evaluation in wireless multi-hop networks using multiplayer games, in: Proceedings of NetGames, 2003.

[60] A. P. Jardosh, E. M. Belding-Royer, K. C. Almeroth, S. Suri, Real-world environment models for mobile network evaluation, IEEE Journal on Selected Areas in Communications 23 (3) (2005) 622-632.

[61] D. Batacharjee, A. Rao, C. Shah, M. Shah, A. Helmy, Empirical modeling of campuswide pedestrian mobility: Observation on the USC campus, in: IEEE Vehicular Technology Conference (VTC), 2004.

[62] M. McNett, G. M. Voelker, Access and mobility of wireless PDA users, Tech. Rep. CS2004-0780, UC San Diego (2004).

[63] N. Aschenbruck, M. Frank, P. Martini, J. Tolle, Human mobility in MANET disaster area simulation - a realistic approach, in: 29th Annual IEEE International Conference on Local Computer Networks (LCN'04), 2004.

[64] S. Ray, Realistic mobility for MANET simulation, Master's thesis, The University of British Columbia (2003).

[65] L. Hogie, F. Guinand, P. Bouvry, The madhoc metropolitan adhoc network simulator, Available at http://www-lih.univ-lehavre.fr/ hogie/madhoc (2005).

[66] K. Maeda, K. Sato, K. Konishi, A. Yamasaki, A. Uchiyama, H. Yamaguchi, K. Yasumoto, T. Higashino, Getting urban pedestrian flow from simple observation: Realistic mobility generation in wireless network simulation, in: Proceedings of the 8th ACM/IEEE International Symposium on Modeling, Analysis and Simulation of Wireless and Mobile Systems(MSWiM2005), 2005, pp. 151-158.

[67] M. Musolesi, C. Mascolo, A community based mobility model for ad hoc network research, Tech. Rep. CS-UCL Research Note RN/05/31, University College London (2005).

[68] B. D. Hankin, R. A. Wright, Passenger flow in subways, operations research quarterly 9 (1958) 81-88.

[69] M. Schreckenberg, S. D. Sharma, Pedestrian and Evacuation Dynamics, springer verlag, 2002.

[70] J. Fruin, Pedestrian Planning and Design, Metropolitan association of urban designer and evironmental planners, 1971.

[71] D. Helbing, Verkehrsdynamik: Nueu Physikalische Modellierungskonzept, Springer Verlag, Berlin, 1997.

[72] F. Navin, R. J. Weeler, Pedestrian flow characteristics, Traffic Engineering.

[73] On fluid mechanics of human crowd motion, Transportation Research 8 (1974) 509-515.

[74] A. Hanisch, T. Schulze, Online simulation of pedestrian flow in public buildings, in: S. Chick, P. J. Sánchez, D. Ferrin, D. J. Morrice (Eds.), Proceedings of the 2003 Winter Simulation Conference, 2003. 
[75] M. Florian, M. Mahut, N. Tremblay, A hybrid optimization-mesoscopic simulation dynamic traffic assignment model, in: Proceedings of the 2001 IEEE Intelligent Transport Systems Conference, 2001.

[76] V. J. Blue, J. L. Adler, Cellular automata microsimulation of bi-directional pedestrian flows, Journal of the Transportation Research Board 1678 (2000) 135-141.

[77] A. Schadschneider, Bionics-inspired cellular automaton model for pedestrian dynamics, in: Traffic and Granular Flow '01, Springer, 2002.

[78] J. Dijkstra, A. Jessurun, H. Timmermans, A multi-agent cellular automata model of pedestrian movement, in: M. Schreckenberg, S. Sharma (Eds.), Pedestrian and Evacuation Dynamics, Springer-Verlag, Berlin, 2001, pp. 173-181.

[79] T. Schelhorn, D. O'Sullivan, M. Haklay, M. Thurstain-Goodwin, STREETS: An agentbased pedestrian model, Tech. Rep. 9, Center for advanced spatial analysis working paper, center for (1999).

[80] B. Jiang, Multi-agent simulations for pedestrian crowds, in: A. Bargiela, E. Kerckhoffs (Eds.), European Simulation Symposium and Exhibition, Nottingham, 1998, pp. 383387.

[81] K. Teknomo, Y. Takeyama, H. Inamura, Microscopic pedestrian simulation model to evaluate "lane-like segregation" of pedestrian crossing, in: Proceedings of Infrastructure Planning Conference, 2001.

[82] C. R. Rindt, J. E. Marca, M. G. McNally, Toward dynamic, longitudinal, agent-based microsimulation models of human activity in urban settings, in: TRB 2003 Annual Meeting, 2003.

[83] F. Tecchia, C. Loscos, R. Conroy, Y. Chryanthou, Agent behavior simulator (ABS): A platform for urban behavior development, in: Proc. Game Technology, 2001.

[84] K. Ashida, S.-J. Lee, J. Allbeck, H. Sun, N. Badler, D. Metaxas, Pedestrians: Creating agent behaviors through statistical analysis of observation data, in: Computer Animation, 2001.

[85] R. J. Beckman, The transportation analysis simulation systems (TRANSIMS) - the Dallas-Ft. Worth case study, Tech. Rep. LAUR-97-4502LANL, Los Alamos National Laboratory (1997).

[86] A. F. Abdelghany, H. S. Mahmassani, Temporal-spatial micro-assignment and sequencing of travel demand with Activity/Trips chains, in: TRB 2003 Annual Meeting, 2003.

[87] S. P. Hoogendoorn, M. Hauser, N. Rodrigues, Application of microscopic pedestrian flow simulation to station design evaluation in Lisbon train stations, in: TRB 2004 Annual Meeting, 2004.

[88] R. Jayakrishnan, C. E. Cortés, R. Lavanya, L. Pagès, Simulation of urban transportation networks with multiple vehicle classes and services: Classifications, functional requirements and general-purpose modeling schemes, in: TRB 2003 Annual Meeting, 2003.

[89] M. Pursula, Simulation of traffic systems - an overview, Journal of Geographic Information and Decision Analysis 3 (1999) 1-8.

[90] R. AKÇELIK, M. BESLEY, Microsimulation and analytical methods for modelling urban traffic, in: Conference on Advance Modeling Techniques and Quality of Service in Highway Capacity Analysis, Truckee, California, 2001.

[91] FHWA, Traffic flow theory: A state of the art report, Tech. rep., 
http://www.tfhrc.gov/its/tft/tft.htm (1995).

[92] R. Akçelik, M. Besley, Acceleration and deceleration models, in: 23rd Conference of Australian Institutes of Transport Research (CAITR 2001), 2002.

[93] K. Teknomo, Y. Takeyama, H. Inamura, Data collection method for pedestrian movement variables, Journal of Civil Engineering Science and Technology 2 (2000) 43-48.

[94] S. J. Older, The speed, density and flow of pedestrians on footway in shopping streets, Traffic Engineering and Control 10 (1968) 160-163.

[95] L. F. Henderson, The statistics of crowd fluids, nature 229 (1971) 381-383.

[96] M. Batty, Agent-based pedestrian modelling, Tech. Rep. 61, Centre of advanced spatial analysis, University College London (2003).

[97] S. P. Hoogendoorn, P. H. L. Bovy, Pedestrian route-choice andb activity scheduling theory and models, transportation research B.

[98] J. Fruin, Pedestrian Planning and Design, Metropolitan Association of Urban Designers and environmental planners, new york, 1971.

[99] S. A. James, C. M. Walton, Platoon pedestrian movement analysis: A case study utilizing the market street station in denver, colorado, Tech. Rep. SWUTC/00/1675100, Southwest Regional University Transportation Center (2000).

[100] L. E. Owen, Y. Zhang, L. Rao, G. McHale, Traffic flow simulation using corsim, in: Winter Simulation Conference, 2000.

[101] J. G. Jetcheva, Y.-C. Hu, S. P. Chaudhuri, A. K. Saha, D. B. Johnson, Design and evaluation of a metropolitan area multitier wireless ad hoc network architecture, in: Fifth IEEE Workshop on Mobile Computing Systems Applications WMCSA, Monterey, CA, 2003.

[102] E. Huang, W. Hu, J. Crowcroft, I. Wassell, Towards commercial mobile ad hoc network applications: A radio dispatch system, in: Proceedings of the 6th ACM International Symposium on Mobile Ad Hoc Networking and Computing, ACM, Urbana-Champaign, IL, 2005, pp. 355-365.

[103] M. Bechler, W. Franz, L. Wolf, Mobile internet access in FleetNet, in: 13. Fachtagung Kommunikation in Verteilten Systemen (KiVS 2003), 2003. 